\title{
Essai d'évaluation du pouvoir colorant d'une source naturelle de jaunissant (Leucaena leucocephala) sur la pigmentation du jaune d'euf chez la poule
}

\author{
D Zongo ${ }^{1 *}, \mathrm{C} \mathrm{Ba}^{1}$, O Diambra ${ }^{1}$, M Coulibaly ${ }^{2}$ \\ ${ }^{I}$ Département de zootechnie, Ensa, 01 BP 5861, Abidjan; \\ ${ }^{2}$ Lacena, $06 \mathrm{BP} 353$, Abidjan 06, Côte-d'Ivoire
}

(Reçu le 28 novembre 1995 ; accepté le 30 septembre 1996)

\begin{abstract}
Summary - Coloration effect of a natural source of pigment (Leucaena leucocephala) for use in poultry. An experiment was conducted to assess Leucaena's potential for use as xanthophyll source for poultry in the south of Côte d'Ivoire. Three diets were evaluated: i) negative control (without pigment); ii) positive control (with commercial carophyll); and iii) Leucaena. Average dietary energy and protein levels were $2700 \mathrm{kcal} \mathrm{ME} / \mathrm{kg}$ and $15 \%$. Positive control and Leucaena treatments contained $40 \mathrm{ppm}$ (apocaroten ) and $29 \mathrm{ppm}$ (xanthophyll), respectively. The diets were fed by 45 layers used in three treatments of 15 layers in each treatment with three animals per cage. The data indicated that there was no significant difference between treatments in terms of egg production and egg weight. Significant differences were observed only for yolk coloration. However, positive control and Leucaena diet scores were comparable $(P<0.05)$.
\end{abstract}

Leucaena leucocephala / layer diets / yolk coloration / tropics

Résumé - Le pouvoir colorant d'une source naturelle de pigments (Leucaena leucocephala) sur le jaune d'œuf a été étudié à partir de trois régimes : i) témoin négatif (sans jaunissement); ii) témoin positif (avec un jaunissant commercial) ; iii) Leucaena. Les régimes contenaient en moyenne 2700 kcal d'énergie métabolisable par kilo et $15 \%$ de matières azotées. Les pigments ont été incorporés à raison de 40 et $29 \mathrm{ppm}$, respectivement pour l'apocarotène (Rousselot) et les xanthophylles du Leucaena. Quarante-cinq pondeuses, réparties en batterie à raison de trois par cage, ont reçu ces régimes pendant 2 mois. Les résultats indiquent que les valeurs moyennes du nombre d'œufs pondus et du poids moyen de l'œuf n'étaient pas significativement différents $(p>0,05)$. En revanche, les notes moyennes de coloration étaient significativement différentes entre les traitements; tandis que celles du témoin positif et du Leucaena étaient comparables $(p<0,05)$.

Leucaena leucocephala / aliment pondeuse / coloration du jaune d'euf / milieu tropical

* Correspondance et tirés à part 


\section{INTRODUCTION}

La coloration du jaune d'œuf est due à des pigments appelés caroténoïdes qui sont habituellement deux xanthophylles: la lutéine et la zéaxanthine. La teneur en pigments du jaune est presque uniquement fonction de ceux inclus dans le régime : c'est l'alimentation et elle seule qui la conditionne. Les sources naturelles de pigments sont, selon Scott et al (1982), Sauveur (1988) et Ba (1989), la farine verte de luzerne $(250 \mathrm{mg})$, le maïs jaune ( 20 à $25 \mathrm{mg} / \mathrm{kg}$ ), les pétales de soucis et d'œillets d'Inde $(6000$ à $10000 \mathrm{mg} / \mathrm{kg}$ ), les algues (2 200 à $4000 \mathrm{mg} / \mathrm{kg})$, le piment et le Leucaena $(440$ à $660 \mathrm{mg} / \mathrm{kg}$ ), la cola, l'huile de palme, le sorgho, etc. Toutefois, les doses d'incorporation permettant $d$ 'atteindre des résultats intéressants se heurtent encore à des problèmes pratiques et économiques. En outre, ces pigments naturels sont difficiles à extraire et, de surcroît, sujets à des pertes par oxydation et isomérisation lors de la conservation et des traitements technologiques (Livingston et al, 1968 ; Davies, 1976 ; Moss et Weedon, 1976). Les processus technologiques qui pourraient préserver une farine à haute teneur en xanthophylles sont très importants si la farine doit être utilisée comme un agent jaunissant. Bailey et Chen (1988) ont en effet montré que chez le cynodon (Cynodon dactylon) on peut préserver $97 \%$ des caroténoïdes et $94 \%$ des xanthophylles par lyophilisation contre seulement environ 40 et $50 \%$ respectivement par séchage à l'étuve.

Deux caroténoïdes de synthèse ont été testés sur les poulets de chair et pour la pigmentation du jaune de l'œuf. La canthaxantine, qui est un pigment rouge, permet d'obtenir des poulets de chair avec une peau jaune orangée. Les doses d'incorporation sont de 2 à $10 \mathrm{~g}$ par tonne d'aliment. L'ester de l'apocarotène, à raison de 2 à $8 \mathrm{~g}$ par tonne d'aliment, permet d'obtenir des cufs bien colorés. Ces produits de synthèse sont autorisés en alimentation animale. Cepen- dant, bien que supposés atoxiques, ils sont interdits dans certains pays ( $S$ cott et al, 1982).

La couleur du jaune d'œuf est un critère important auquel s'attache le consommateur bien qu'elle ne préjuge en rien de la valeur nutritionnelle du produit (Sauveur, 1988). Sous peine cependant de voir l'œuf se déprécier sur le marché, le producteur doit trouver les moyens pour améliorer la couleur du jaune avec les pigments disponibles.

Qu'il s'agisse de pigments naturels ou artificiels, les agents de pigmentation du jaune d'œuf sont importés à des prix prohibitifs par les fabricants d'aliments de poules pondeuses de Côte-d'Ivoire et de nombreux autres pays tropicaux en développement. Face à cette situation, et étant donné que ces pays produisent très peu de maïs jaune, il nous est apparu utile d'évaluer le potentiel de coloration d'une substance naturelle locale riche en pigments : le Leucaena leucocephala.

\section{MATÉRIEL ET MÉTHODES}

\section{Matériel végétal}

\section{Le Leucaena leucocephala}

Le genre Leucaena appartenant à la famille des légumineuses (mimosacées) compte 13 espèces, dont l'aire d'origine s'étend du Pérou au Texas. Sa culture est de plus en plus répandue dans les tropiques, l'espèce la plus connue étant Leucaena leucocephela, aux petites fleurs blanches groupées à l'extrémité des rameaux (von Maydell, 1983 ; Brewbacker, 1987). L leucocephala est un arbre qui produit des aliments pour l'homme, du fourrage pour le bétail, du bois de feu transformable en charbon, du bois d'œuvre et de construction, de l'engrais vert pour les champs, etc (Maydell, 1983). Il a une bonne valeur alimentaire et contient aussi de la mimosine, qui diminuerait la thyroxine sérique et en limiterait l'utilisation. 


\section{Techniques et méthodes d'étude}

\section{Préparation de la farine de feuilles \\ de Leucaena et extraction des xanthophylles}

Les feuilles de L leucocephala, fraîchement récoltées à la ferme de l'École nationale supérieure agronomique (Ensa) d'Abidjan, ont été séchées à l'étuve à l'obscurité à $70^{\circ} \mathrm{C}$ pendant $48 \mathrm{~h}$ et moulues dans un broyeur Brabender LS munie d'une grille avec des pores de $1 \mathrm{~mm}$ de diamètre. Cette farine est stockée dans un sac gardé à l'abri de la lumière et à la température ambiante jusqu'à la date d'utilisation. Les xanthophylles du Leucaena ont été extraites à l'aide d'une solution hexane/acétone $(7 / 3, \mathrm{~V}: \mathrm{V})$, évaporée à sec. Les résidus repris dans $25 \mathrm{~mL}$ d'éther de pétrole pur (Naumann et Bassler, 1976) ont servi à l'évaluation quantitative par élution à l'éthanol sur un chromatographe liquide à haute performance au Laboratoire central de nutrition animale d'Abidjan.

\section{Expérimentation}

Quarante-cing pondeuses de la souche Harco, âgées de 18 semaines, ont été réparties en trois traitements (T1-, T2 + et T3) de 15 poules chacun avec cinq répétitions de trois animaux par répétition (cage).

Les caractéristiques des aliments distribués sont indiquées dans le tableau I. Les quantités de pigments pour $1 \mathrm{~kg}$ d'aliment étaient de 40 ppm (apocarotène/carophylle) et de 29 ppm (xanthophylles/Leucaena) respectivement pour les aliments témoin positif (carophylle) et Leucaena. Les aliments et l'eau étaient servis à volonté. Chaque poule recevait $100 \mathrm{~g}$ d'aliment par jour. Ces quantités étaient totalement consommées par les trois groupes de poules.

L'essai s'est déroulé à la ferme de l'Ensa et a duré 8 semaines.

\section{Mesures effectuées}

À partir de la $21^{\mathrm{e}}$ semaine, chaque jour, les œufs collectés ont été dénombrés et pesés. Pour chacun des traitements, ont été retenus trois œufs qui ont été identifiés, pesés et conservés pendant $20 \mathrm{j}$ dans une salle où la température moyenne, au cours des 2 mois, était de $28 \pm 2{ }^{\circ} \mathrm{C}$.
La couleur du jaune des œufs a été appréciée par comparaison à une série de couleurs étalons, celles de l'échelle Roche. Cette échelle a été établie expérimentalement selon les normes du système trichromatique élaborées par la Commission internationale de l'éclairage (Anonyme, 1975). Elle se présente sous la forme d'un éventail à 15 branches numérotées de 1 à 15 et qui correspondent à des intensités croissantes de coloration. Une note de 1 dénote un jaune très pâle et une note de 15 indique une couleur jaune orangée très accentuée.

Tous les $20 \mathrm{j}$, les œufs ont été repris, cassés et deux examinateurs ont évalué l'un après l'autre la couleur du jaune en donnant une note. La note retenue était la moyenne arithmétique des notes des deux examinateurs.

\section{Analyses statistiques}

Toutes les données ont été traitées par l'analyse de la variance et les valeurs moyennes comparées par la méthode de Newman-Keuls (Dagnélie, 1975).

\section{RÉSULTATS ET DISCUSSION}

Les résultats concernant le nombre d'œufs, le poids moyen de l'cuf et les notes de coloration sont regroupés dans le tableau II.

Le nombre moyen d'cufs pondus et le poids moyen de l'œuf obtenus dans les trois traitements sont comparables. Sur la base des densités nutritives des régimes et du rationnement réalisé, les calories et les protéines ingérées étaient équivalentes dans les trois traitements. On note, en revanche, des différences significatives $(p<0,05)$ en ce qui concerne la note de coloration entre les traitements renfermant les xanthophylles et le témoin négatif. Les scores du jaunissant commercial et du Leucaena ne présentent aucune différence, alors que le premier renferme 40 ppm d'apocarotène et le second 29 ppm de pigments non identifiés par kilo. On sait que lorsque la température est relativement élevée l'absorption de nourriture baisse et que l'activité pigmentaire est inhibée par la teneur en calcium de l'aliment. 
Tableau I. Caractéristiques des aliments distribués.

\begin{tabular}{|c|c|c|c|}
\hline \multirow[b]{3}{*}{$\begin{array}{l}\text { Taux d'incorporation } \\
\text { des xanthopylles }\end{array}$} & \multicolumn{3}{|c|}{ Traitements } \\
\hline & $\begin{array}{l}\text { Témoin négatif } \\
\text { sans jaunissant }\end{array}$ & $\begin{array}{c}\text { Témoin positif } \\
\text { carophylle } \\
\text { jaune/rouge }\end{array}$ & $\begin{array}{l}\text { Leucaena } \\
\text { leucocephala }\end{array}$ \\
\hline & 0 & $40 \mathrm{ppm}$ & $29 \mathrm{ppm}$ \\
\hline \multicolumn{4}{|c|}{ Composition centésimale (\%) } \\
\hline Maïs & 65,16 & 65,16 & 65,16 \\
\hline Farine de poisson & 9,69 & 9,69 & 9,69 \\
\hline Tourteaux de coprah & 10,18 & 10,16 & 4,16 \\
\hline Remoulage de blé & 5,00 & 5,00 & 5,00 \\
\hline Huile de palme & 2,00 & 2,00 & 2,00 \\
\hline $\mathrm{CaCO}_{3}$ & 7,27 & 7,29 & 7,29 \\
\hline $\mathrm{NaCl}$ & 0,20 & 0,20 & 0,20 \\
\hline Prémix ${ }^{1}+$ carophylle & - & 0,5 & - \\
\hline Farine de Leucaena & - & - & 6 \\
\hline \multicolumn{4}{|c|}{ Valeurs nutritives calculées ${ }^{2}$} \\
\hline EM (Mcal/kg) & 2,72 & 2,71 & 2,79 \\
\hline Protéines brutes (\%) & 15,10 & 14,66 & 15,10 \\
\hline Cellulose brute $(\%)$ & 4,33 & 3,33 & 4,00 \\
\hline Matière grasse (\%) & 5,30 & 8,80 & 5,00 \\
\hline Extractif non azoté (\%) & 52,77 & 56,11 & 55,00 \\
\hline Cendres $(\%)$ & 11,40 & 10,30 & 10,30 \\
\hline Calcium $(\%)$ & 3,50 & 3,75 & 3,75 \\
\hline Phosphore total (\%) & 0,62 & 0,59 & 0,59 \\
\hline Potassium (\%) & 0,39 & 0,39 & 0,32 \\
\hline Sodium $(\%)$ & 0,22 & 0,2 & 0,18 \\
\hline
\end{tabular}

\footnotetext{
${ }^{1}$ Le prémix contient (par kilo) : vit A, 500000 UI ; Vit D3, 50000 UI; Vit E, $500 \mathrm{mg}$; Vit B1, $80 \mathrm{mg}$; Vit B2, $60 \mathrm{mg}$; Vit B6, $120 \mathrm{mg}$; Vit B12, $400 \mathrm{mg}$; acide folique, $32 \mathrm{mg}$; Vit pp, $720 \mathrm{mg}$; Vit B3, $240 \mathrm{mg}$; choline, $400 \mathrm{mg}$; Vit K3, $1000 \mathrm{mg}$; Mn, $6000 \mathrm{mg}$; flavomycine, $100 \mathrm{mg}$; $1,20 \mathrm{mg}$; Cu, $20 \mathrm{mg}$; Zn, 4000 $\mathrm{mg} ; \mathrm{Fe}, 2400 \mathrm{mg}$; Co, $10 \mathrm{mg}$; carophylle (apocarotène) : $8000 \mathrm{mg}$. ${ }^{2}$ Calculs basés sur NRC (1984).
}

C'est pourquoi on relève les teneurs en calcium et en caroténoïdes en climat chaud (Anonyme, 1972). Nos résultats indiquent que les xanthophylles contenus dans les feuilles de Leucaena ont un pouvoir jaunis- sant indéniable. En attendant des études complémentaires à l'instar de celles déjà réalisées sur d'autres supports naturels (Yokoyama et White, 1965 ; Hartel et Ostendorf,1971), et compte tenu de la présence 
Tableau II. Pouvoir colorant de la farine de Leucaena leucocephala sur la pigmentation du jaune de l'œuf de poule.

\begin{tabular}{lccc}
\hline & \multicolumn{3}{c}{ Traitements } \\
\cline { 2 - 4 } Paramètres & $\begin{array}{c}\text { Témoin négatif } \\
\text { sans jaunissant }\end{array}$ & $\begin{array}{c}\text { Témoin positif } \\
\text { carophylle } \\
\text { jaune/rouge }\end{array}$ & $\begin{array}{c}\text { Leucaena } \\
\text { leucocephala }\end{array}$ \\
\hline Nombre moyen d'œufs & $48 \mathrm{a}(8,22)$ & $41 \mathrm{a}(5,75)$ & $41 \mathrm{a}(5,01)$ \\
Poids moyen de l'œuf $(\mathrm{g})$ & $55,84 \mathrm{a}(1,20)$ & $54,10 \mathrm{a}(0,23)$ & $55,78 \mathrm{a}(1,19)$ \\
Note de coloration & $3,61 \mathrm{a}(1,69)$ & $6,81 \mathrm{~b}(2,71)$ & $6,78 \mathrm{~b}(2,45)$ \\
\hline
\end{tabular}

Les valeurs moyennes sur la même ligne avec les mêmes lettres ne sont pas significativement différentes $(p>0,5)$. Entre parenthèses : écart type.

dans le Leucaena de mimosine, acide aminé toxique, il importe de réserver les rameaux feuillus de cet arbuste aux ruminants qui en sont friands.

\section{CONCLUSION}

Il apparaît, au terme de ce travail, que l'on dispose sous les tropiques d'une source potentielle naturelle de jaunissant pour la pigmentation du jaune d'œuf chez la poule : le Leucaena leucocephala, légumineuse arbustive connue depuis longtemps pour ses multiples usages tant en agriculture qu'en élevage. Cependant, du fait de sa teneur en mimosine, il est souhaitable que des recherches ultérieures, fondées sur un protocole amélioré soient envisagées pour extraire, purifier, tester et préciser les effets réels et les limites éventuelles d'utilisation du Leucaena comme agent de pigmentation du jaune d'œuf.

\section{RÉFÉRENCES}

Anonyme (1972) La cithranaxanthine, agent de pigmentation du jaune d'œuf. Doc BASF Aktiengesellschaft, D-6700 Ludwigshafen
Anonyme (1975) La pigmentation du jaune de l'œuf. Document Hoffman-La Roche $n^{\circ} 1347$ Neuillysur-Seine, France

Ba C (1989) Évaluation de deux sources naturelles pour la pigmentation de l'œuf : Leucaena et piment rouge. Mémoire DEA, Univ Nat de Côte-d'Ivoire, Abidjan

Bailey CA, Chen BH (1988) Carotene and xanthophyll changes during growth and processing of turf Bermudagrass. Poultry Sci 67,1644-1646

Brewsbaker JL (1987) Leucaena leucocephala: $a$ decade of development (Steppler HA, Nair PKR, eds) ICRAF, Nairobi, 289-323

Dagnélie P (1975) Théorie et méthodes statistiques. Vol 2. Presses agronomiques, Gembloux, Belgique, $2^{\mathrm{e}}$ édition, 245-250

Davies BU (1976) Caretenoids. In: Chemistry and Biochemistry of plant pigments (Godwin TW, ed) Vol 2. Academic Press, New York, NY, 38-165

Hartel H, Ostendorf S (1971) Taux d'assimilation de la lutéine et de la zéaxanthine provenant des supports naturels pigmentaires (maiis jaune, mais " plata ", gluten de maïs et farine verte d'herbe) chez les poules pondeuses. Kraftfutter 24, 420-423

Livingston AL, Knowles RE, Nelson JW, Kohler GO (1968) Xanthophyll and carotene loss during pilot and industrial scale alfalfa processing. J Agric Food Chem 16, 84-87

Maydell von HJ (1983) Arbres et arbustes du Sahel. Leurs caractéristiques et leurs utilisations. $\mathrm{N}^{\circ} 147$, Édition GTZ, 293-294

Moss GP, Weedon BCL (1976) Chemistry of the carotenoids. In: Chemistry and biochemestry of plant pigments (Godwin TW, ed) Vol 2. Academic Press, New York, NY, 149-219 
National Research Council (NRC) (1984) Nutrient requirements of poultry. National Academy Press, 2101 Constitution Ave, NW, Washington, DC 20 418, USA

Naumann K, Bassler R (1976) Die chemische Untersuchung von futermitteln. Methodenbuch-band III.

Sauveur B (1988) Reproduction des volailles et production d'oufs. Inra, Paris, 377-435
Scott ML, Nesheim MC, Young RJ (1982) Nutrition of chicken. ML Scott and Associates publishers, Ithaca, NY

Yokoyama H, White MI (1965) Caroténoïdes des agrumes. Il. Structure de la citranaxanthine, un nouveau caroténoïde-cétone. J Org Chem 30, 24812482 